\title{
Olfactory aversive conditioning alters olfactory bulb mitral/tufted cell glomerular odor responses
}

\author{
Max L. Fletcher *t \\ Department of Neurobiology and Anatomy, University of Texas Medical School, Houston, TX, USA
}

Edited by:

Federico Bermudez-Rattoni, Universidad Nacional Autónoma de México, Mexico

\section{Reviewed by:}

Takashi Yamamoto, Osaka University Graduate School of Dentistry, Japan

Raúl G. Paredes, National University of Mexico, Mexico

\section{*Correspondence:}

Max L. Fletcher, Department of Anatomy and Neurobiology, University of Tennessee Health Science Center, 855 Monroe Avenue, Memphis, TN 38163, USA. e-mail:mfletch4@uthsc.edu

tPresent address:

Department of Anatomy and Neurobiology, University of Tennessee Health Science Center, Memphis, TN, USA.
The anatomical organization of receptor neuron input into the olfactory bulb (OB) allows odor information to be transformed into an odorant-specific spatial map of mitral/tufted $(\mathrm{M} / \mathrm{T})$ cell glomerular activity at the upper level of the OB. In other sensory systems, neuronal representations of stimuli can be reorganized or enhanced following learning. While the mammalian OB has been shown to undergo experience-dependent plasticity at the glomerular level, it is still unclear if similar representational change occurs within (M/T) cell glomerular odor representations following learning. To address this, odorant-evoked glomerular activity patterns were imaged in mice expressing a GFP-based calcium indicator (GCaMP2) in OB (M/T) cells. Glomerular odor responses were imaged before and after olfactory associative conditioning to aversive foot shock. Following conditioning, we found no overall reorganization of the glomerular representation. Training, however, did significantly alter the amplitudes of individual glomeruli within the representation in mice in which the odor was presented together with foot shock. Further, the specific pairing of foot shock with odor presentations lead to increased responses primarily in initially weakly activated glomeruli. Overall, these results suggest that associative conditioning can enhance the initial representation of odors within the $\mathrm{OB}$ by enhancing responses to the learned odor in some glomeruli.

Keywords: olfactory learning, associative conditioning, odor map, glomerulus, GCaMP2

\section{INTRODUCTION}

All olfactory receptor neurons (ORNs) expressing the same receptor protein project their axons to specific glomeruli in the olfactory bulb $(\mathrm{OB})$ where they form excitatory synapses onto a circuit of diverse cell types including mitral/tufted (M/T) cells and several types of juxtaglomerular interneurons (Buck and Axel, 1991; Ressler et al., 1994; Kiyokage et al., 2010). The convergence of receptor input onto $\mathrm{OB}$ output neurons forms the basis of an odorant-specific spatial map of $\mathrm{M} / \mathrm{T}$ cell glomerular activity in which each glomerulus represents the molecular receptive range of the ORN type that innervates it (Mori et al., 2006).

In other sensory systems, learning can often enhance or shift cortical maps of sensory information following learning (Gilbert et al., 2001). Similarly, experiments in the rodents have shown that $\mathrm{OB}$ glomerular layer processing of odor information is altered with learning as well. For example, associative conditioning in rat pups leads to increased 2-DG glomerular uptake (Coopersmith et al., 1986; Sullivan and Leon, 1986; Woo et al., 1987; Woo and Leon, 1991) and increased c-fos expression in juxtaglomerular neurons and deeper layer granule cells in regions responding to the trained odorant (Johnson et al., 1995; Funk and Amir, 2000; Salcedo et al., 2005). Together these experiments point toward learning leading to long-term changes in glomerular layer interneuronal responses to learned odorants.

While numerous studies have reported changes in M/T cell firing following learning (Wilson et al., 1985; Buonviso and Chaput, 2000; Ravel et al., 2003; Fletcher and Wilson, 2003; Doucette and Restrepo, 2008; Doucette et al., 2011), comparatively little is known about changes in $\mathrm{M} / \mathrm{T} \mathrm{OB}$ spatial representations of odorants following learning. Work from the Freeman lab has also shown that the EEG spatial pattern measured across the bulb for a given odor changes following associative conditioning to that odorant (Freeman and Schneider, 1982; Grajski and Freeman, 1989). More recently, olfactory associative conditioning has also been shown to increase $\mathrm{OB}$ dorsal surface intrinsic signal responses to the trained odor (Yuan et al., 2002). While these studies clearly demonstrate long-term changes in both the temporal and spatial patterns of M/T odor responses following learning, it is still not fully clear how $\mathrm{M} / \mathrm{T}$ cell glomerular odor representations are affected in mammals following associative learning.

Optical imagining in insects however, has recently revealed that olfactory associative conditioning can enhance glomerular level projection neuron representations to the trained odor (Faber et al., 1999; Yu et al., 2004). For example, in bees, pairing an odorant with a sucrose reward lead to increases in the response amplitude of only the glomeruli within the trained odor representation (Faber et al., 1999). While in Drosophila, aversive conditioning has been shown to lead to a recruitment of previously unresponsive glomeruli into the trained odorant glomerular representation (Yu et al., 2004).

In this study, we tested whether aversive associative conditioning alters $\mathrm{M} / \mathrm{T}$ cell glomerular odor representations in the mammalian $\mathrm{OB}$, and whether the enhanced is driven by new glomeruli 
recruited into the odor representation or altered responses from existing glomeruli. This was accomplished issue using transgenic mice that express the fluorescent $\mathrm{Ca}^{2+}$ indicator protein GCaMP2 exclusively in $\mathrm{OB} \mathrm{M} / \mathrm{T}$ cells with no expression in inhibitory interneurons. In these mice, odor-evoked glomerular activity patterns that reflect $\mathrm{M} / \mathrm{T}$ cell glomerular responses can be visualized on the dorsal surface with individual glomerular resolution (Fletcher et al., 2009). Optical imaging in these transgenic mice allow the unique advantage of comparing glomerular responses from the same glomeruli before and after training in the same animal. Through multi-day optical imaging, glomerular odor responses were compared before and after olfactory associative conditioning with the same odorant. The results suggest that associative conditioning enhances glomerular representation to the trained odor primarily by increasing responses in initially weakly activated glomeruli within the representation.

\section{MATERIALS AND METHODS ANIMALS AND SURGERY}

Adult transgenic mice expressing the fluorescent calcium indicator protein GCaMP2 under the Kv3.1 potassium channel promoter were used (Diez-Garcia et al., 2005). In these mice, GCaMP2 is expressed primarily in $\mathrm{OB}$ output neurons, M/T cells, with no expression in olfactory sensory neuron axons or OB inhibitory interneurons (Fletcher et al., 2009). Odor-evoked changes in glomerular fluorescence in GCaMP2 mice therefore, reflect glomerular level $\mathrm{M} / \mathrm{T}$ cell odor responses.

For surgery and imaging, mice were anesthetized with sodium pentobarbital $(50 \mathrm{mg} / \mathrm{kg}$, i.p.). Mice were secured in a custom stereotaxic apparatus (Narishige) and a chronic imaging window was made on dorsal surface of the skull by thinning the bone overlying the OBs. The imaging window was covered with a thin layer of cyanoacrylate glue (Maxicure, BSI, Inc.) to improve optical transparency and seal the skull. An anchor screw (\#000-120, 1/16" length, Small Parts, Inc.) was inserted into the parietal bone. A head post made of 16-gauge syringe needle tubing was placed on top of the skull and secured into place with acrylic dental cement (Stoelting). In some animals, the clarity of optical imaging window significantly degraded overtime due to surgical damage or problems with the glue adhering to the surface of the skull. In both cases, these animals were removed from the study. All animal care protocols were approved by the University of Texas and the University of Tennessee Institutional Animal Care and Use Committees.

\section{ASSOCIATIVE CONDITIONING}

Mice were placed into four groups: a group that received pairings of odor and foot shock (Paired, $n=10$ ), a group that only received foot shocks (Shock only, $n=6$ ), and group that received only odorant presentations (Odor only, $n=6$ ), and a group with no exposure to either MV or foot shock (Naïve, $n=4$ ). The conditioning apparatus consisted of a small plexiglas cylinder placed inside a standard mouse test cage equipped with a floor of parallel steel bars connected to a precision animal shocker (Coulbourn Instruments). Associative conditioning took place in a single session in which the mice received 10 trials of $1 \% \mathrm{vol} / \mathrm{vol}$ methyl valerate diluted in mineral oil (MV) (4s duration) terminating in $0.25 \mathrm{~s}$ duration, $0.4 \mathrm{~mA}$ foot shocks. The conditions for the control groups were identical except the mice received either 10 trials of shock only ( $0.25 \mathrm{~s}$ duration, $0.4 \mathrm{~mA})$ or 10 trials $\mathrm{MV}$ only (4s duration). All trials were separated by an inter-trial interval of $2 \mathrm{~min}$. On the day following training, animals were placed in a two-odor choice test. This test involved placing an animal into a standard mouse cage that contained a small cotton swab containing the MV on one side of the cage and a small cotton swab containing a novel odor (peppermint) on the other. Mice were placed along the midline of the cage between the two odorant zones and allowed to freely move around the cage for $60 \mathrm{~s}$. Each animal received three, $60 \mathrm{~s}$ trials $(180 \mathrm{~s}$ total) in which the amount of time spent on each side of the cage was recorded. MV was chosen as the paired odorant because it activates a cluster of glomerular on the dorsal surface that can be easily accessed (Figure 2).

\section{OPTICAL IMAGING AND ANALYSIS}

Imaging was performed on an Olympus BX50WI microscope using a $10 \times(0.3 \mathrm{NA})$ Olympus objective. OBs were illuminated by a monochromator centered at $480 \mathrm{~nm}$ (Polychrome II, TILL Photonics GmbH). GCaMP2 fluorescence was band-pass filtered with a Chroma emission filter (HQ535/50) and collected using a CCD camera at $25 \mathrm{~Hz}$ (NeuroCCD-SM256, Redshirt Imaging). All animals were imaged on three sessions spaced days apart. The first two sessions (Days 1-4) took place prior to conditioning with all mice naive to the odor. On Day 7, mice received one of the three conditioning protocols. On the following day (Day 8), mice were imaged again. During each session, MV was presented 8-10 times. Glomerular odor representations were generated from the averages of the individual trials on each day.

Maps of odor-evoked spatial activity were generated by first correcting for photobleaching and then spatially low-pass filtered as described previously (Fletcher et al., 2009). For each trial, the odor-evoked change in fluorescence was calculated by subtracting the average of five frames immediately preceding stimulus onset from the average of five frames centered on the peak of the response from the first respiration for each pixel. Images reflecting the relative change in fluorescence $(\Delta \mathrm{F} / \mathrm{F})$ were then calculated by dividing the odor-evoked change in fluorescence by the resting fluorescence. The final normalized odor map for each trial was generated by dividing the entire image by the mean response of all activated glomeruli. For each day, all normalized trials were averaged and represented in pseudo color to give the mean normalized response for that day. For pre- versus post-training comparisons, odor maps were overlaid and re-aligned using skull landmarks and blood vessel patterns.

For quantitative analysis, mean normalized individual glomerular responses for each day were calculated from the $\Delta \mathrm{F} / \mathrm{F}$ response measured at the center of each glomerulus $(3 \times 3$ pixel average), normalized to the mean of all glomerular responses, and average across all trials. All individual glomerular responses were calculated from the raw data without any spatial filtering. A glomerulus was considered to be activated if its odor-evoked signal amplitude was five-times greater than the rms noise level calculated from an adjacent non-responsive area (Fletcher et al., 2009). While individual glomeruli could be identified in the same 
animal across days, with our limited odor set we were unable to identify the same glomerulus across animals. Due to this, all group analysis was limited to comparing the relative responses of the glomeruli as a population. For comparisons across days, the mean absolute difference $(\mathrm{MAD})=\left\langle\left|x_{1}-x_{2}\right|\right\rangle$, where $x_{1}$ and $x_{2}$ are the normalized $\Delta \mathrm{F} / \mathrm{F}$ responses measured on the first and second imaging sessions for each glomerulus was calculated (Kent and Mozell, 1992; Verhagen et al., 2007).

To compare changes in the MV odor representation as a whole, each day's responses from all glomeruli from each animal was represented in a multidimensional odor space (Deisig et al., 2006). The MV representation for each day was expressed as a vector in in $n$-dimensional odor space where each dimension is represented by the responses from each individual glomerulus. To compare changes in the MV representations across days, we calculated their proximity in odor space by comparing their Euclidian distances. The Euclidian distance between the MV representations for each day was calculated as:

$$
d_{i j}=\sqrt{\sum_{k=1}^{p}\left(X_{i}-X_{j}\right)^{2}}
$$

with $i$ and $j$ indicating days, $p$ the number of glomeruli, and $X_{i}$ the response of glomerulus $k$ on day $i$. For comparisons across groups, the mean Euclidian distance between the MV representations on D1 and D4 and D4 and D8 were compared. As no manipulations occurred between D1 and D4 in any group, the Euclidian distances should be similar across groups and represent the day-to-day variability in the representations. We then compared this mean Euclidian distance to the distances between D4 and D8 representations that spanned the training day. For visualization, we then used principle component analysis followed by a varimax rotation to project the representations from each group into a three dimensional odor space. Together these three factors accounted for over $97 \%$ of the variance in each group.

During all imaging sessions, animals were freely breathing and respiratory activity was monitored with a piezoelectric device strapped around the chest. Respiratory rates were taken from random trials of each imaging session for each animal. In some sessions, the piezoelectric device did not reliably detect respiration. In these animals, the respiratory rate was calculated from the respiratory oscillation observed in the odor-evoked GCaMP2 odor-evoked signal. Overall, the mean respiratory rate across all animals and days was $1.72 \pm 0.06 \mathrm{~Hz}$ (range: $1.0-3.1 \mathrm{~Hz}$ ).

\section{ODORANT PRESENTATION}

During imaging, methyl valerate was delivered using a flowdilution olfactometer previously described (Fletcher et al., 2009). Separate flow controllers for the clean air and the pure odorant vapor were used to mix the flow streams at the end of the odor delivery system to achieve an approximate concentration of $0.25 \%$ s.v. at a flow rate of $0.7 \mathrm{~L} / \mathrm{min}$. Odor pulse duration was $2 \mathrm{~s}$ with an inter-stimulus interval of at least $60 \mathrm{~s}$ to prevent any short-term adaptation of the responses. For behavioral training, MV was presented through a small hole at the top of the chamber at a flow rate of $1 \mathrm{~L} / \mathrm{min}$ using an olfactometer (Vulintus). For the behavioral training and testing, pure odorants were dissolved in mineral oil to a $1 \% \mathrm{vol} / \mathrm{vol}$ concentration. This concentration of MV was used for training and testing to account for diffusion of the odorant in the behavioral chamber. The intensity of the odorant in the behavioral chamber was similar to the intensity of the MV delivered in the imaging experiments.

\section{RESULTS}

To confirm that the training paradigm used here leads to odor learning, a subset of the imaged mice was placed in a two-odor choice behavioral task on D8 before the final imaging session. Mice in the Paired group spent significantly less time on the MV side compared to naïve non-imaged mice, shock-only, or odoronly mice, suggesting a learned MV aversion (percent time on MV side: Naïve: $46.7 \pm 2.1 \%$; Shock Only: $51.6 \pm 1.3 \%$; Odor Only: $50.0 \pm 5.5 \%$; Paired: $30.6 \pm 3.4 \%$; ANOVA), Main effect of day: $\left[F_{(2,60)}=4.78, p=0.03\right]\left(\right.$ ANOVA, $\left[F_{(3,18)}=7.57, p<0.05\right]$; post-hoc Fisher's test, $p<0.05$ ) (Figure 1).

Mice were imaged on D1 and D4 to establish stable baseline responses and imaged again following training (D8). Based on D1 responses from all animals (22 mice, 327 glomeruli), MV activated an average of $14.9 \pm 3.6$ glomeruli located on the anterior-dorsal region of the $\mathrm{OB}$ (Figure 2). The average raw odor response of all glomeruli per animal decreased slightly across days for all three groups, with all groups showing similar decreases (Means: Day $1=5.31 \% \Delta \mathrm{F} / \mathrm{F}$; Day $4=4.55 \% \Delta \mathrm{F} / \mathrm{F}$; Day $8=$ $4.42 \% \Delta \mathrm{F} / \mathrm{F})$ ANOVA, Main effect of day: $\left[F_{(2,60)}=4.78, p=\right.$ $0.03]$. Despite this, no significant decrease was observed in any group following training (D4-D8 comparisons; post hoc Fisher's tests: Shock Only: $p=0.61, n s$; Odor Only: $p=0.22$, $n s$; Paired: $p=0.73, n s)$.

During imaging, normalized responses remained stable across the first two sessions (Dayl and Day4) with the number

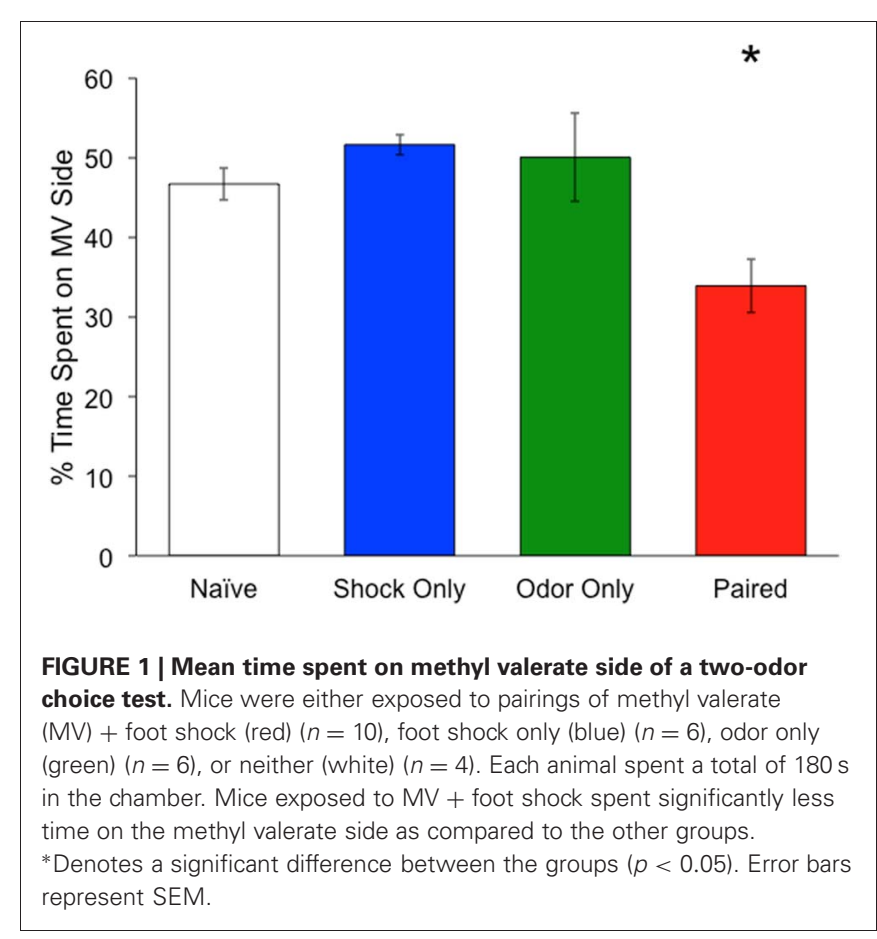




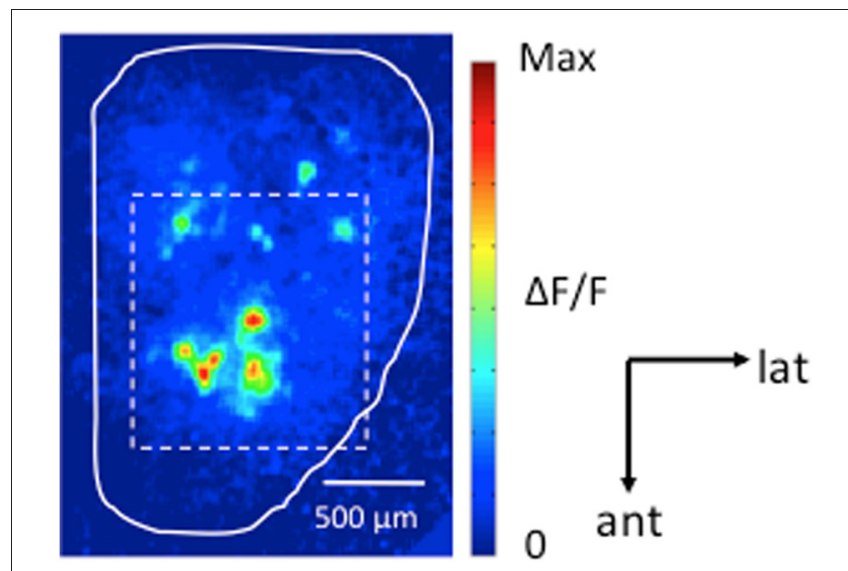

FIGURE 2 | Odor-evoked G-CaMP2 $\mathrm{Ca}^{2+}$ responses imaged in the olfactory bulb. Dorsal view of a pseudo-color response map induced by methyl valerate $(0.25 \%$ s.v.). The visible region of the dorsal bulbar surface under $10 \times$ magnification is outlined by the dashed white line. Across all animals ( $n=22$ mice, 327 glomeruli), $0.25 \%$ MV activated an average of $14.9 \pm 3.6$ glomeruli located on the anterior-dorsal region of the $\mathrm{OB}$ with an average amplitude of $5.0 \pm 1.6 \% \Delta \mathrm{F} / \mathrm{F}$. Scale bar: $500 \mu \mathrm{m}$.

and position of activated glomeruli identical for all groups [Figures 1A, 2A, and 3A (Day1-Day4)]. Similar results were obtained following training, with the number of glomeruli remaining stable [Figures $\mathbf{3 A}, \mathbf{4 A}$, and $\mathbf{5 A}$ (Day4-Day8)]. However, changes in normalized glomerular responses to $\mathrm{MV}$ were observed in the Paired group (Figure 5A). In this case, the relative amplitudes of a few glomeruli within the representation were observed to increase in response to MV.

To further explore this, we obtained a measure of the mean day-to-day change in glomerular responses prior to any odor training by calculating the mean D1-D4 percent change (Shock Only group: $0.49 \pm 10.97 \%$; Odor Only group: $-0.12 \pm 9.71 \%$; Paired group: $1.66 \pm 14.11 \%)$, All groups combined: $0.92 \pm$ $12.37 \%$, mean $\pm 1 \mathrm{SD})$. We found no differences in mean D1-D4 percent change between groups ANOVA, $\left[F_{(3,326)}=0.972, p=\right.$ $0.41]$. Because no training took place during the first two imaging sessions, we used the overall mean D1-D4 percent $\pm 2 \mathrm{SD}$ as a range of normal day-to-day variability in glomerular odor responses. Thus, any responses falling out of this range $(-23.82-$ $25.66 \%$, Figures 3B, 4B, 5B, gray boxes) could be considered significant changes. For the examples shown here, no post-training responses were found to be outside of this range in either of the mice receiving only foot shocks or presentations of $\mathrm{MV}$ alone (Figures 4B and 5B). However, in the mouse receiving pairings of $\mathrm{MV}$ and foot shock, two glomeruli showed significant posttraining response increases (Figure 5B, glomeruli \#1 and \#8), and once glomerulus showed a significant post-training response decrease (Figure 5B, glomerulus \#19).

In accord with this, we observed more large post-training increases (D4-D8 percent change $\geq 25 \%$ ) from glomeruli in the Paired group (9.3\%) than either the Shock only $(3.2 \%)$ or Odor only group $(4.1 \%)$. The percentage of glomeruli displaying significant post-training response decreases was small for all groups (Shock Only: 1.1\%; Odor Only: 1.4\%; Paired: 1.9\%). The same

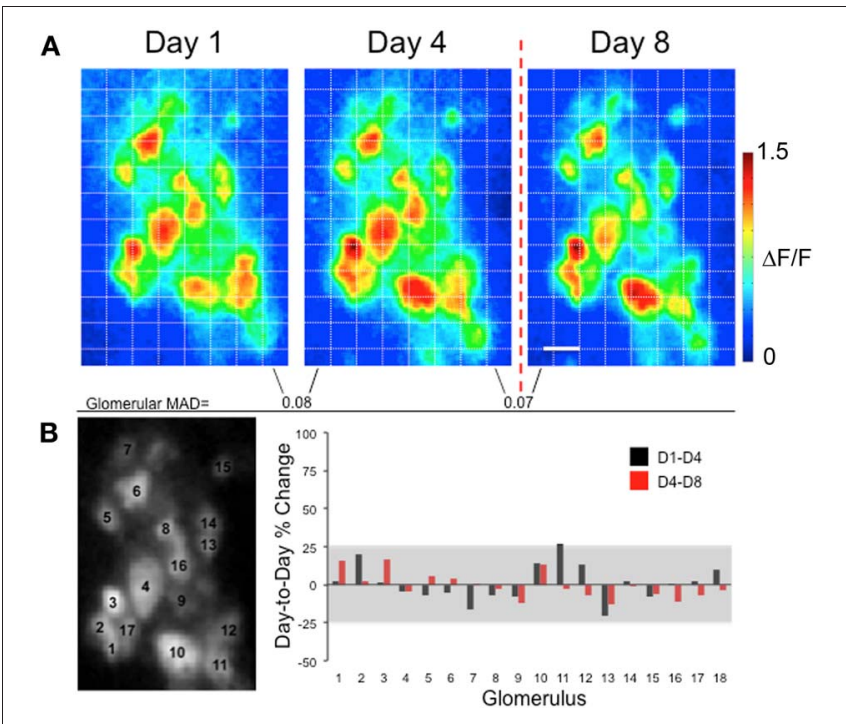

FIGURE 3 | Presentations of foot shock alone do not significantly alter glomerular G-CaMP2 odor representations in individual mice.

(A) Methyl valerate response maps taken from the same animal across several days. Maps from Day 1 to Day 4 were taken prior to any conditioning and were similar across the days. The last map (Day 8) was taken one day after exposing the animal to presentations of shock only. No dramatic changes in response amplitude were seen in glomeruli on the day following the foot shock. The Mean Absolute Day-to-Day Difference (MAD) for each glomerulus remained similar before and after training. Scale bar: $100 \mu \mathrm{m}$ (B) The left panel indicates the position of the glomeruli. The graph shows the percent change in response for each glomerulus across days. The gray box represents the pre-training percent change (mean $\pm 2 S D$ ) taken from all glomeruli across all three groups. As can be seen in this example, the pre- and post-training changes in individual glomerular responses, while variable, did not exceed this value.

trend was observed when the cutoff for percent change was lowered to the mean D1-D4 percent change $\pm 1 \mathrm{SD})$.

To quantify response differences across the glomerular population for each group, the mean day-to-day absolute difference in glomerular response (MAD) for each animal was calculated. This measure gives the mean change in response across all glomeruli. In this analysis, the lower the MAD, the more similar the responses. Since glomerular responses were measured on two separate days prior to training, a comparison of the responses from these days gave a baseline measure of day-to-day variation in responses. The pre-training (D1-D4) MAD was found to be similar across all groups (MAD: Shock only: $0.08 \pm 0.006 \% \Delta \mathrm{F} / \mathrm{F}$; Odor only: $0.09 \pm 0.005 \% \Delta \mathrm{F} / \mathrm{F}$; Paired: $0.09 \pm 0.008 \% \Delta \mathrm{F} / \mathrm{F}$ )

(Figure 6A, black bars). These values were similar to the mean trial-to-trial MAD calculated from consecutive odor trials taken from each animal on each day $(\mathrm{MAD}=0.09 \pm 0.01 \% \Delta \mathrm{F} / \mathrm{F})$ (Figure 6B).

To compare changes in glomerular responses following training, the glomerular responses taken from the last pre-training day (D4) and the day following the training (D8) were used to calculate the post-training (D4-D8) MAD for each group (MAD: Shock only: $0.08 \pm 0.003 \% \Delta \mathrm{F} / \mathrm{F}$; Odor only: $0.08 \pm$ $0.005 \% \Delta \mathrm{F} / \mathrm{F}$; Paired: $0.14 \pm 0.007 \% \Delta \mathrm{F} / \mathrm{F}$ ) (Figure 6A, red bars). In comparing the pre- and post-training MAD, a significant 


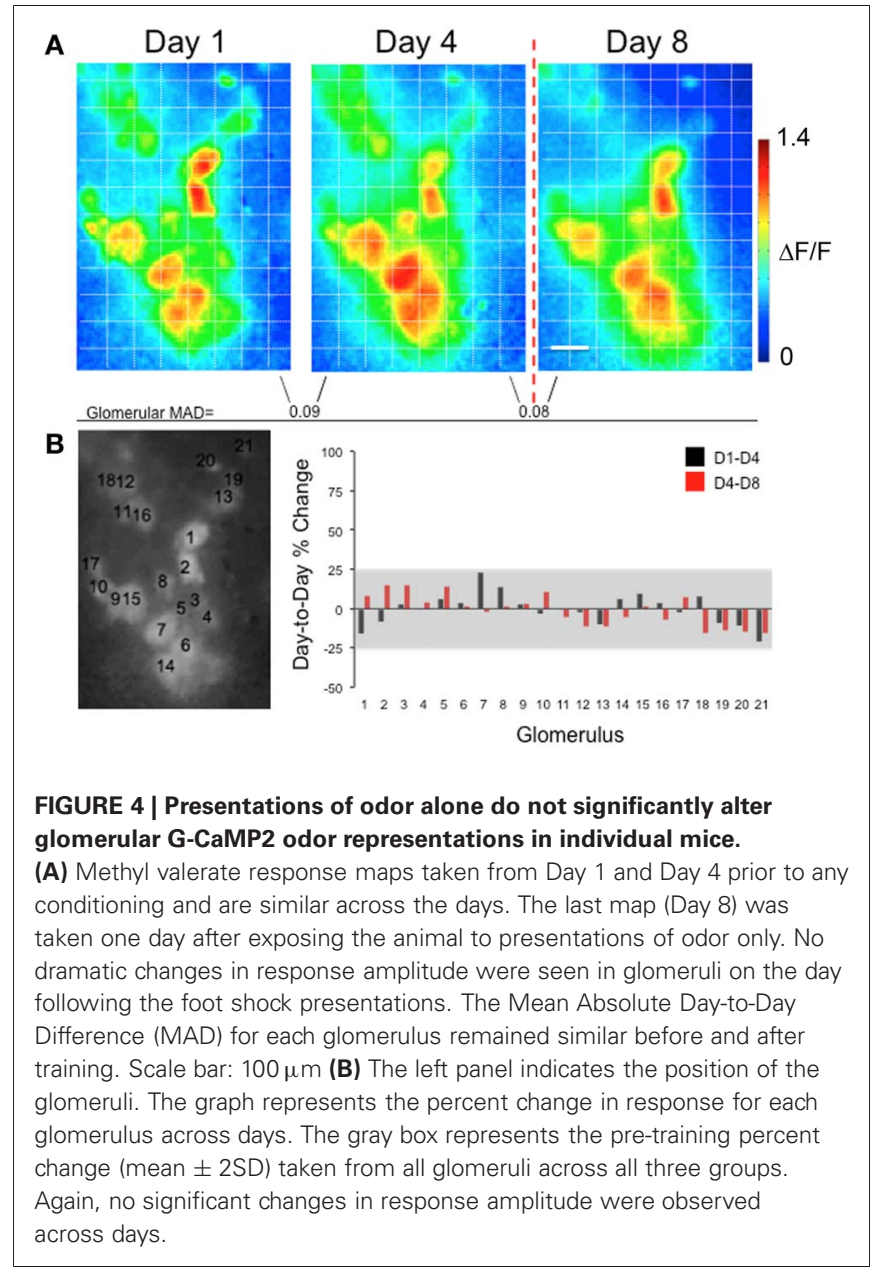

difference between the three groups was found ANOVA: main effect of group: $\left[F_{(2,44)}=4.27, p<0.05\right]$, with the Paired group displaying a significant increase between the mean pre- and posttraining MAD (post hoc Fisher's test, $p<0.05$ ). This effect was not observed in the other groups.

Within the Paired group, we noticed that the largest posttraining changes were often observed in the initially weaker responding glomeruli. To quantify this, we divided all the glomeruli in each group into quartiles based on their initial responses to $\mathrm{MV}$ and compared the average post-training percent change in each quartile. In both the Shock only and Odor only groups, average post-training percent changes were small in each quartile (Figure 7). Additionally, no significant differences were observed across quartiles in either group (Shock only: ANOVA: $\left[F_{(3,90)}=0.48, p=0.7\right]$; Odor only: ANOVA: $\left[F_{(3,70)}=2.36\right.$, $p=0.08]$. However, in the Paired group, we found that a significant increase in the post-training percent change in glomeruli from the lowest quartile of initial response (Paired: ANOVA: $\left[F_{(3,90)}=7.84, p<0.05\right.$; post hoc Fisher's test, $\left.p<0.05\right]$. In line with this, we observed more large post-training increases (D4-D8 percent change $\geq 25 \%$ ) from first quartile glomeruli in the Paired group $(41.7 \%)$ than either the Shock only $(0 \%)$ or Odor only group (9\%).
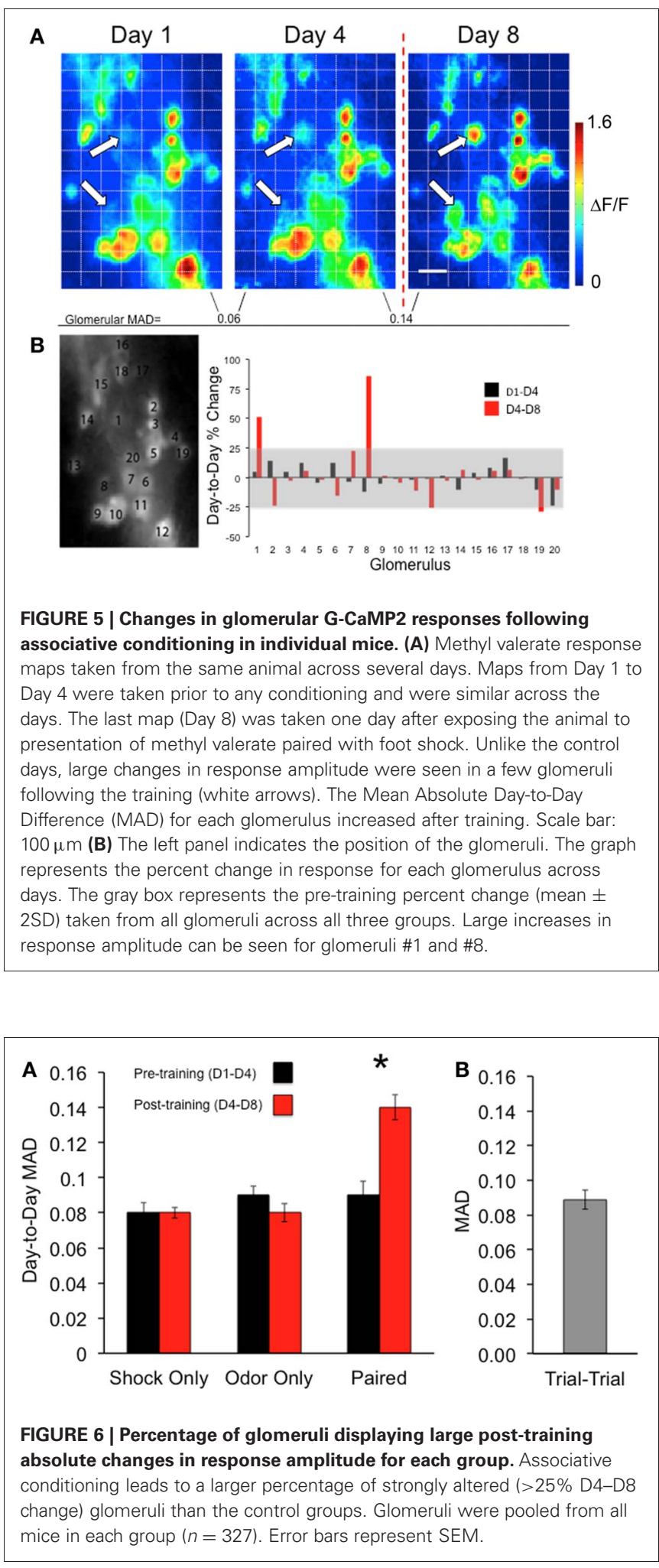

To investigate whether the changes in individual glomerular responses following training are enough to alter the overall representation of the odor, we compared the Euclidian distances in putative olfactory space between the D1 and D4 


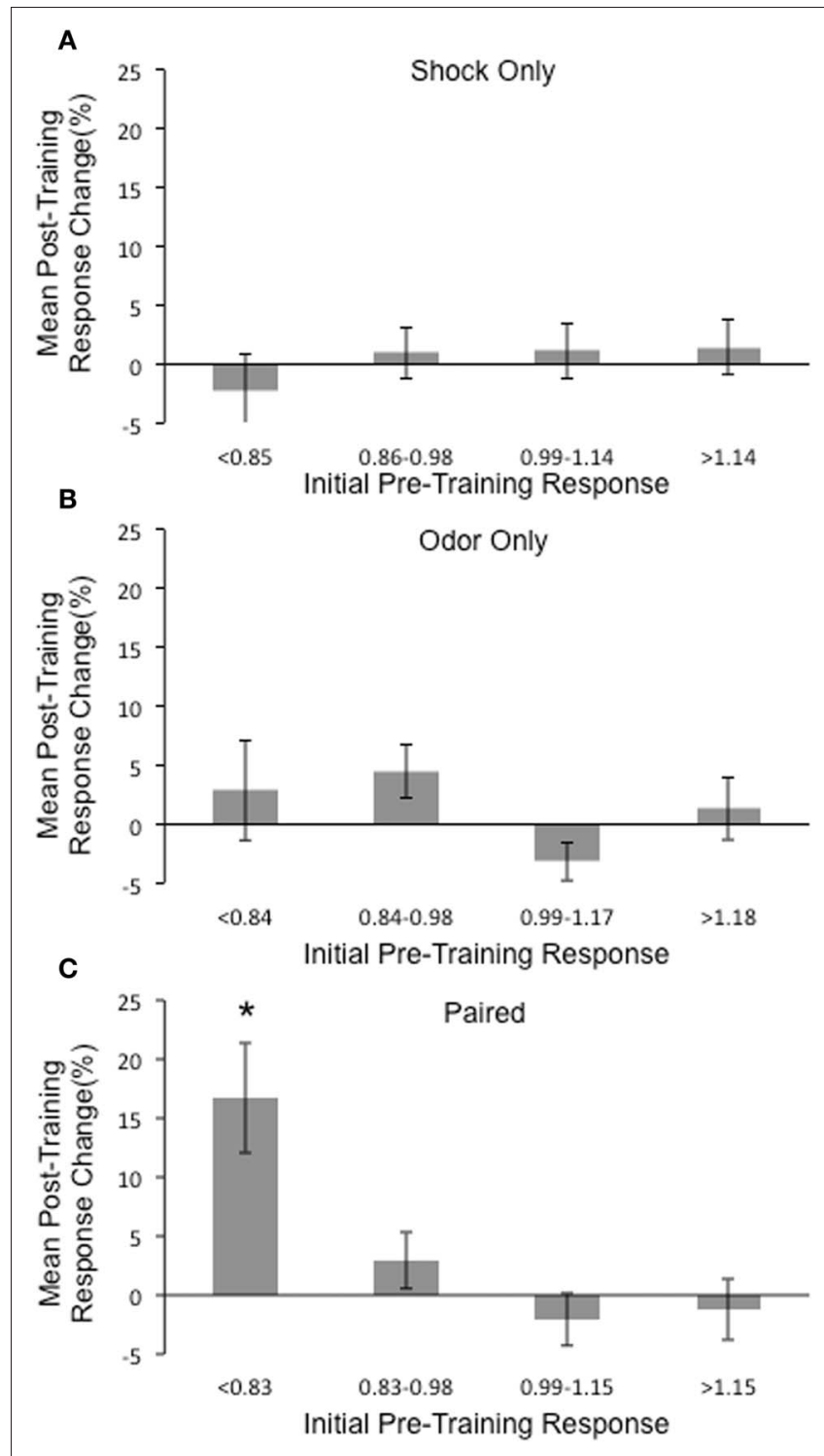

FIGURE 7 | Comparison of the mean post-training response changes in glomeruli based on initial responses to MV. All glomeruli from each group $(n=327)$ were divided into quartiles based on their initial normalized responses to $\mathrm{MV}$ and the average post-training percent change was calculated for each quartile. (A) No significant mean post-training changes were observed in any quartile for the Shock only group. (B) No significant mean post-training changes were observed in any quartile for the Odor only group. (C) A significant increase in the post-training percent change in glomeruli from the lowest quartile of initial response in glomeruli from the Paired group was found. *Denotes a significant difference between the groups $(p<0.05)$.

representations to that between the D4 and D8 representations in each animal. As no manipulations occurred between D1 and D4 in any group, the Euclidian distances were similar across groups (Shock only $=0.40$; Odor only $=0.32$; Paired $=$ 0.45). Following the training, only the Paired group showed a shift in the representation as measured by the Euclidian distance (Shock only $=0.44$; Odor only $=0.34$; Paired $=0.66$ ). Paired $t$-tests revealed a significant difference only in the Paired group (Shock only: $t_{(5)}=-0.98, p=0.37$; Odor only: $t_{(5)}=$ -0.61, $p=0.56$; Paired: $t_{(5)}=-2.40, p<0.05$ ) (Figure 8A). To visualize this shift in the representation, we performed PCA analysis on the all the glomerular responses from each group across days. MV representations from each group were projected onto a 3-dimensional odor space based on the first three principle components (Figures 8B-D). As can be seen in the two control groups, the post-training D8 odor representations are clustered close to the D1 and D4 responses. However, in the Paired group only, the D8 response shifted away from the D1 and D4 responses.

\section{DISCUSSION}

Using in vivo imaging, this study investigated the impact of olfactory aversive learning on $\mathrm{OB} \mathrm{M} / \mathrm{T}$ cell glomerular odor responses in adult mice. The results demonstrate that olfactory learning significantly alters $\mathrm{OB} \mathrm{M} / \mathrm{T}$ cell glomerular odor representations to the trained odor by altering the response amplitudes of glomeruli within the representation. Overall, we find that associative conditioning alters $\mathrm{M} / \mathrm{T}$ cell responses following training primarily by increasing the responses in the initially weakest responding glomeruli. Overall, this study provides further evidence that the neuronal representations of odors that predict aversive or emotional events can be enhanced at the first synapses of central olfactory processing. These changes are potentially large enough to alter the representation of the odorant at the glomerular level and could serve as the basis for learning-induced changes in higher olfactory centers.

These findings expand upon previous studies reporting enhanced glomerular responses following learning in rodents (Coopersmith et al., 1986; Johnson et al., 1995; Yuan et al., 2002; Salcedo et al., 2005). In these studies, subsequent exposure to the trained odorant lead to increased c-fos activation in populations of juxtaglomerular neurons (Johnson et al., 1995; Salcedo et al., 2005; Funk and Amir, 2000), as well as increased 2-deoxyglucose uptake in glomerular layer neurons in regions known to be activated by the learned odorant (Woo et al., 1987; Woo and Leon, 1991). Since the calcium indicator used is this study is expressed only in M/T cells (Fletcher et al., 2009), the changes that we observed in glomerular responses reflect changes in $\mathrm{M} / \mathrm{T}$ cell glomerular tuft responses to OSN input. Our results suggest that aversive conditioning enhances $\mathrm{M} / \mathrm{T}$ cell glomerular responses in some glomeruli and that this enhancement is driven largely by the weakest responding glomeruli of the representation. As calcium increases measured within a glomerulus reflect a population $\mathrm{M} / \mathrm{T}$ cells, the increased responses following learning seen in some glomeruli could reflect increases in individual M/T cell activity, recruitment of additional $\mathrm{M} / \mathrm{T}$ cells associated with the glomerulus, or both. Similar results have been reported in insects, with associative conditioning leading to enhanced principle neuron activity into the antennae lobe glomerular representation of the trained odorant (Faber et al., 1999; Daly et al., 2004; Yu et al., 2004). However, unlike similar studies in Drosophila where previously unresponsive glomeruli were recruited into the odor representation following associative conditioning (Yu et al., 2004), we found no instances of recruitment of new glomeruli following conditioning. 

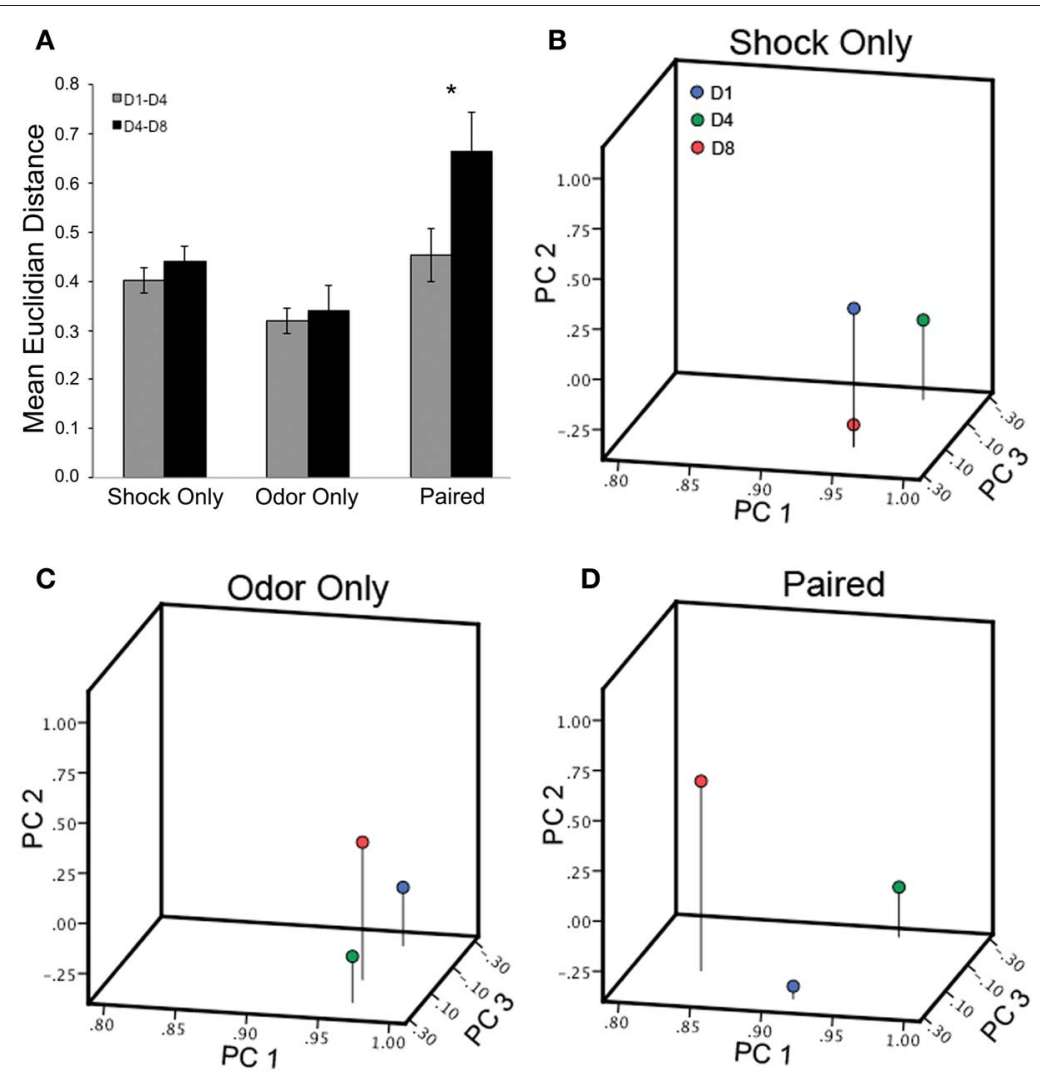

FIGURE 8 | Olfactory associative conditioning significantly alters the representation of the trained odor in odor space. (A) Euclidian distances in putative olfactory space between the D1 and D4 representations and between the D4 and D8 representations were compared for each group. Following the training, the only the Paired group showed a shift in the representation as measured by the Euclidian distance. (B-D) MV representations in odor space before and after conditioning in all three groups (Day 1: blue; Day 4: green; Day 8: red). In both the Shock only and Odor only groups, the post-training D8 odor representations are clustered close to the D1 and D4 responses. However, in the Paired group, the D8 response has shifted away from the D1 and D4 responses. *Denotes a significant difference between the groups $(p<0.05)$.
While we did observe some glomeruli in the Paired animals that significantly increased, we did not observe any large-scale changes in response amplitude across all glomeruli following learning. This is in contrast to earlier studies in rat pups that have reported an overall decrease in odor-evoked M/T cell responses following associative conditioning (Wilson et al., 1985; Wilson and Leon, 1988). Unlike these studies, we found very few instances of decreases in $\mathrm{M} / \mathrm{T}$ glomerular odor responses following learning. There are several potential reasons for this difference. First, increased fluorescence in calcium indicators observed in $\mathrm{M} / \mathrm{T}$ dendritic glomerular tufts reflects a combination of calcium influx from OSN-driven activation of NMDA receptors, dendritic-initiated spiking, and back-propagating somaticinitiated action potentials (Chen et al., 2002; Yuan et al., 2004; Zou et al., 2006; Fletcher et al., 2009). It is likely that the learninginduced increases in $\mathrm{M} / \mathrm{T}$ cell odor-evoked suppression reported in the earlier electrophysiological studies are driven by changes in deeper granule cell mediated inhibition (Brennan and Keverne, 1997). Given the strong calcium signals observed in M/T dendritic tufts in response to OSN input (Chen et al., 2002; Zou et al., 2006), it is possible that this deeper level inhibition in $\mathrm{M} / \mathrm{T}$ cell output is masked when only observing activity in the tuft. Another potential explanation lies in the fact that the studies mentioned above (Wilson et al., 1985; Wilson and Leon, 1988) were performed in rat pups and our study was performed in adult mice. As it is clear that infants rodents possess a unique and immature olfactory system (Greer et al., 1982; Raineki et al., 2010), it is possible that the olfactory learning in infants and adults utilizes different mechanisms. Finally, it is important to note that our technique only allows us to observe glomerular odor representations located across the dorsal surface. 2-DG mapping studies in mice have shown that methyl valerate activates a cluster of glomeruli in the posterior-lateral region of the $\mathrm{OB}$ as well (Johnson et al., 2009). With our technique, we can only observe the glomerular representation on the dorsal surface and we may be missing the effects of training on glomeruli located in other regions.

\section{POTENTIAL MECHANISMS OF GLOMERULAR PLASTICITY}

As $\mathrm{M} / \mathrm{T}$ cell odor responses reflect a combination of input from sensory neurons, interneuronal inhibition, and neuromodulatory input from centrifugal cortical and neuromodulatory regions (Kosaka and Kosaka, 2005; Fletcher and Chen, 2010; Kiyokage et al., 2010), one possible mechanism behind this effect could 
involve Hebbian plasticity at the level of ORN input into the bulb (Ennis et al., 1998). In this case, it is possible that the simultaneous activation of OB neurons by ORN input and centrifugal activation via foot shock creates the conditions necessary to induce long-term changes in the sensitivity of the ORN-M/T cell synapse.

Additionally, it is possible that the enhanced glomerular responses following associative conditioning could be caused by learning-dependent structural changes within the glomerular layer. For example, associative conditioning has also been shown to increase the size of glomeruli responsive to the trained odorant (Woo et al., 1987; Jones et al., 2008). In one case, this size increase appears to be due to increases in the number of ORN axons innervating the glomeruli (Jones et al., 2008). While interesting, it is unlikely that similar anatomical reorganization is driving the increased responses seen in glomeruli in this study, as the changes observed in these studies required several days of associative conditioning and our study look at responses the day immediately following training.

\section{POTENTIAL IMPACT ON ODOR DISCRIMINATION AND PERCEPTION}

The post-training enhancement of glomerular response seen here could have several consequences on M/T cell odor coding.

\section{REFERENCES}

Brennan, P. A., and Keverne, E. B. (1997). Neural mechanisms of mammalian olfactory learning. Prog. Neurobiol. 51, 457-481.

Buck, L., and Axel, R. (1991). A novel multigene family may encode odorant receptors, a molecular basis for odor recognition. Cell 65, 175-187.

Buonviso, N., and Chaput, M. (2000). Olfactory experience decreases responsiveness of the olfactory bulb in the adult rat. Neuroscience 95, 325-332.

Chen, C. F., Barnes, D. C., and Wilson, D. A. (2011). Generalized versus stimulus-specific learned fear differentially modifies stimulus encoding in primary sensory cortex of awake rats. J. Neurophysiol. 106, 3136-3144.

Chen, W. R., Shen, G. Y., Shepherd, G. M., Hines, M. L., and Midtgaard, J. (2002). Multiple modes of action potential initiation and propagation in mitral cell primary dendrite. $J$. Neurophysiol. 88, 2755-2764.

Coopersmith, R., Lee, S., and Leon, M. (1986). Olfactory bulb responses after odor aversion learning by young rats. Brain Res. 389, 271-277.

Daly, K. C., Christensen, T. A., Lei, H., Smith, B. H., and Hildebrand, J. G. (2004). Learning modulates the ensemble representations for odors in primary olfactory networks. Proc. Natl. Acad. Sci. U.S.A. 101, 10476-10481
Deisig, N., Giurfa, M., Lachnit, H., Sandoz, J. (2006). Neural representation of olfactory mixtures in the honeybee olfactory lobe. Eur. J. Neurosci. 24, 1161-1174.

Diez-Garcia, J., Matsushita, S., Mutoh, H., Nakai, J., Ohkura, M., Yokoyama, J., Dimitrov, D., and Knöpfel, T. (2005). Activation of cerebellar parallel fibers monitored in transgenic mice expressing a fluorescent $\mathrm{Ca} 2+$ indicator protein. Eur. J. Neurosci. 22, 627-635.

Doucette, W., Gire, D. H., Whitesell, J., Lucero, M. T., and Restrepo, D. (2011). Associative cortex features in the first olfactory brain relay station. Neuron 69, 1176-1187.

Doucette, W., and Restrepo, D. (2008). Profound context-dependent plasticity of mitral cell responses in olfactory bulb. PLoS Biol. 6:e258. doi: 10.1371/journal.pbio.0060258

Edeline, J. M., Pham, P., and Weinberger, N. M. (1993). Rapid development of learning-induced receptive field plasticity in the auditory cortex. Behav. Neurosci. 107, 539-551.

Ennis, M., Linster, C., AroniadouAnderjaska, V., Ciombor, K., and Shipley, M. T. (1998). Glutamate and synaptic plasticity at mammalian primary olfactory synapses. Ann. N.Y. Acad. Sci. 855, 457-466.

Faber, T., Joerges, J., and Menzel, R. (1999). Associative learning modifies neural representations of odors

First, the increased responses following training could serve to increase sensitivity in the weaker glomeruli, possibly decreasing behavioral detection thresholds. Second, enhanced glomerular responses may lead to a shift in glomerular odor receptive fields (ORFs). In the auditory system, neuronal RFs can be shifted away from their preferred frequency and toward the paired frequency following tone-foot shock pairings (Lennartz and Weinberger, 1992; Edeline et al., 1993). Similarly, odorant experience has been shown to shift M/T cell ORFs toward the experienced odor (Fletcher and Wilson, 2003). Together these changes could lead to a decorrelation of the representation of the trained odor and other similar odorants, possibly making the trained odor more discriminable. In line with this, behavioral discrimination experiments in both rats and humans studies have demonstrated increased olfaction acuity following associative conditioning (Rabin, 1988; Fletcher and Wilson, 2002; Linster et al., 2002; Li et al., 2008).

\section{ACKNOWLEDGMENTS}

The author thanks Johannes Richter for help with the initial imaging and behavioral experiments, Drs. Wei Chen and Shin Nagayama for helpful discussions, and Dr. Thomas Knöpfel for providing the transgenic mice. The work was supported by NIH grant DC009853 to Max L. Fletcher.

in the insect brain. Nat. Neurosci. 2 74-78.

Fletcher, M. L., and Chen, W. R. (2010). Neural correlates of olfactory learning: critical role of centrifugal neuromodulation. Learn. Mem. 17, 561-570.

Fletcher, M. L., Masurkar, A. V., Xing, J., Imamura, F., Xiong, W., Nagayama, S., Mutoh, H., Greer, C. A., Knopfel, T, and Chen, W. R. (2009). Optical imaging of postsynaptic odor representation in the glomerular layer of the mouse olfactory bulb. J. Neurophysiol. 102, 817-830.

Fletcher, M. L., and Wilson, D. A (2002). Experience modifies olfactory acuity, acetylcholine-dependent learning decreases behavioral generalization between similar odorants. J. Neurosci. 22, RC201.

Fletcher, M. L., and Wilson, D. A (2003). Olfactory bulb mitral-tufted cell plasticity: odorant-specific tuning reflects previous odorant exposure. J. Neurosci. 23, 6946-6955.

Freeman, W. J., and Schneider, W. (1982). Changes in spatial patterns of rabbit olfactory EEG with conditioning to odors. Psychophysiology 19, 44-56.

Funk, D., and Amir, S. (2000) Enhanced fos expression within the primary olfactory and limbic pathways induced by an aversive conditioned odor stimulus. Neuroscience 98, 403-406.

Gilbert, C. D., Sigman, M., and Crist, R. E. (2001). The neural basis of perceptual learning. Neuron 31, 681-697.

Grajski, K. A., and Freeman, W. J. (1989). Spatial EEG correlates of nonassociative and associative olfactory learning in rabbits. Behav. Neurosci. 103, 790-804.

Greer, C. A., Stewart, W. B., Teicher, M. H., and Shepherd, G. M. (1982). Functional development of the olfactory bulb and a unique glomerular complex in the neonatal rat. J Neurosci. 2, 1744-1759.

Johnson, B. A., Woo, C. C., Duong, H., Nguyen, V., and Leon, M. (1995). A learned odor evokes an enhanced Fos-like glomerular response in the olfactory bulb of young rats. Brain Res. 699, 192-200.

Johnson, B. A., Xu, Z., Ali, S. S., and Leon, M. (2009). Spatial representations of odorants in olfactory bulb of rats and mice: similarities and differences in chemotopic organization. J. Comp. Neurol. 514, 658-673.

Jones, S. V., Choi, D. C., Davis, M. and Ressler, K. J. (2008). Learningdependent structural plasticity in the adult olfactory pathway. $J$. Neurosci. 28, 13106-13111.

Kent, P. F., and Mozell, M. M. (1992). The recording of odorantinduced mucosal activity patterns with a voltage-sensitive dye. J. Neurophysiol. 68, 1804-1819.

Kiyokage, E., Pan, Y. Z., Shao, Z., Kobayashi, K., Szabo, G., 
Yanagawa, Y., Obata, K., Okano, H, Toida, K., Puche, A. C., and Shipley, M. T. (2010). Molecular identity of periglomerular and short axon cells. J. Neurosci. 30, 1185-1196.

Kosaka, K., and Kosaka, T. (2005). Synaptic organization of the glomerulus in the main olfactory bulb: compartments of the glomerulus and heterogeneity of the periglomerular cells. Anat. Sci. Int. 80, 80-90.

Lennartz, R. C., and Weinberger, N. M. (1992). Frequency-specific receptive field plasticity in the medial geniculate body induced by pavlovian fear conditioning is expressed in the anesthetized brain. Behav. Neurosci. 106, 484-497.

Li, W., Howard, J. D., Parrish, T. B., and Gottfried, J. A. (2008). Aversive learning enhances perceptual and cortical discrimination of indiscriminable odor cues. Science 319, 1842-1845.

Linster, C., Johnson, B. A., Morse, A., Yue, E., Leon, M. (2002). Spontaneous versus reinforced olfactory discriminations. J. Neurosci. 22, 6842-6845.

Mori, K., Takahashi, Y., Igarashi, K. M., Yamaguchi, M. (2006). Maps of odorant molecular features in the mammalian olfactory bulb. Physiol. Rev. 86, 409-433.

Rabin, M. D. (1988). Experience facilitates olfactory quality discrimination. Percept. Psychophys. 44, 532-540.

Raineki, C., Pickenhagen, A., Roth, T. L., Babstock, D. M., McLean, J. H., Harley, C. W., Lucion, A. B., and Sullivan, R. M. (2010). The neurobiology of infant maternal odor learning. Braz. J. Med. Biol. Res. 43, 914-919.

Ravel, N., Chabaud, P., Martin, C., Gaveau, V., Hugues, E., TallonBaudry, C., Bertrand, O., and Gervais, R. (2003). Olfactory learning modifies the expression of odour-induced oscillatory responses in the gamma $(60-90 \mathrm{~Hz})$ and beta $(15-40 \mathrm{~Hz})$ bands in the rat olfactory bulb. Eur. J. Neurosci. 17, 350-358.

Ressler, K. J., Sullivan, S. L., and Buck, L. B. (1994). Information coding in the olfactory system: evidence for a stereotyped and highly organized epitope map in the olfactory bulb. Cell 79, 1245-1255.

Salcedo, E., Zhang, C., Kronberg, E., and Restrepo, D. (2005). Analysis of training-induced changes in ethyl acetate odor maps using a new computational tool to map the glomerular layer of the olfactory bulb. Chem. Senses 30, 615-626.

Sullivan, R. M., and Leon, M. (1986). Early olfactory learning induces an enhanced olfactory bulb response in young rats. Brain Res. 392, 278-282.

Verhagen, J. V., Wesson, D. W., Netoff, T. I., White, J. A., and Wachowiak,
M. (2007). Sniffing controls an adaptive filter of sensory input to the olfactory bulb. Nat. Neurosci. 10 631-639.

Wilson, D. A., and Leon, M. (1988). Spatial patterns of olfactory bulb single-unit responses to learned olfactory cues in young rats. $J$. Neurophysiol. 59, 1770-1782.

Wilson, D. A., Sullivan, R. M., and Leon, M. (1985). Odor familiarity alters mitral cells response in the olfactory bulb of neonatal rats. Brain Res. 22, 314-317.

Woo, C. C., Coopersmith, R., and Leon, M. (1987). Localized changes in olfactory bulb morphology associated with early olfactory learning. J. Comp. Neurol. 263, 113-125.

Woo, C. C., and Leon, M. (1991). Increase in a focal population of juxtglomerular cell in the olfactory bulb associated with early learning. J. Comp. Neurol. 305, 49-56.

Yu, D., Ponomarev, A., and Davis, R. L. (2004). Altered representation of the spatial code for odors after olfactory classical conditioning; memory trace formation by synaptic recruitment. Neuron 42, 437-449.

Yuan, Q., Harley, C. W., McLean, J. H., and Knöpfel, T. (2002). Optical imaging of odor preference memory in the rat olfactory bulb. J. Neurophysiol. 87, 3156-3159.

Yuan, Q., Mutoh, H., Debarbieux, F., and Knöpfel, T. (2004). Calcium signaling in mitral cell dendrites of olfactory bulbs of neonatal rats and mice during olfactory nerve stimulation and $\beta$-adrenoceptor activation. Learn. Mem. 11, 406-411.

Zou, Z., Xiong, W., Zeng, S., Xia, A., Shepherd, G. M., Greer, C. A., and Chen, W. R. (2006). Dendritic excitability and calcium signaling in the mitral cell distal glomerular tuft. Eur. J. Neurosci. 24 1623-1632.

Conflict of Interest Statement: The author declares that the research was conducted in the absence of any commercial or financial relationships that could be construed as a potential conflict of interest.

Received: 31 January 2012; accepted: 05 March 2012; published online: 22 March 2012.

Citation: Fletcher ML (2012) Olfactory aversive conditioning alters olfactory bulb mitral/tufted cell glomerular odor responses. Front. Syst. Neurosci. 6:16. doi: 10.3389/fnsys.2012.00016

Copyright (c) 2012 Fletcher. This is an open-access article distributed under the terms of the Creative Commons Attribution Non Commercial License, which permits non-commercial use, distribution, and reproduction in other forums, provided the original authors and source are credited. 